\title{
The urea-creatinine ratio as a novel biomarker of critical illness-associated catabolism
}

\author{
Jan Gunst ${ }^{1 *}\left(\mathbb{D}\right.$, Kianoush B. Kashani $^{2}$ (D) and Greet Hermans ${ }^{3}$ (D)
}

(C) 2019 Springer-Verlag GmbH Germany, part of Springer Nature

Despite advances in intensive care medicine, a considerable number of patients evolve into a stage of persistent critical illness, with prolonged dependency on intensive care, which is associated with poor prognosis $[1,2]$. Effectuated by persistent inflammation, neurohumoral alterations, and prolonged immobilization, such patients enter a catabolic state that is often not suppressed by feeding $[3,4]$. Ongoing catabolism ultimately leads to severe muscle wasting and associated weakness, which impairs outcome $[3,5]$. Currently, monitoring catabolism is cumbersome, since there is no routinely available biomarker with acceptable sensitivity and specificity. Assessment of 3-methylhistidine, released into the circulation during protein degradation, is complex and extends beyond routine measurements [6]. Also, accurate monitoring of nitrogen losses and balances poses multiple challenges in critically ill patients [6]. As catabolism is not accurately measured on a routine basis, its presence and severity often become clear once muscle wasting and weakness are established. The lack of validated and routinely available biomarkers of catabolism to some extent hampers epidemiological and interventional studies on this topic.

In this issue of Intensive Care Medicine, Haines et al. identified the urea over creatinine ratio (UCR) as a potential biomarker of critical illness-associated catabolism [7]. In the absence of a gold standard, the evidence supporting the relationship is mainly indirect. Indeed, to confirm their hypothesis, the investigators focused

\footnotetext{
*Correspondence: jan.gunst@kuleuven.be

${ }^{1}$ Clinical Division and Laboratory of Intensive Care Medicine, Department of Cellular and Molecular Medicine, KU Leuven, Herestraat 49, 3000 Leuven, Belgium

Full author information is available at the end of the article
}

on the difference between short- and long-stay intensive care unit (ICU) patients, the latter expectedly being more catabolic. In two large trauma ICU cohorts, involving in total $>4000$ patients, investigators found a significantly different temporal trend in UCR. Although UCR initially increased in all patients, the rise was substantially greater in patients still in the ICU on day 10 as compared to patients discharged alive earlier. After day 9, when the phenotype of persistent critical illness generally has developed [2, 7], daily UCR discriminated patients with prolonged ICU stay better than baseline illness severity and antecedent patient characteristics, putting forward UCR as a key metabolic signature of persistent critical illness. The increase in UCR coincided with a progressive loss of muscle mass, as assessed by L4 psoas and L3 muscle cross-sectional area (CSA) in 107 patients who had serial CT scans available. Based on CSA, patients with persistent critical illness lost muscle mass more profoundly than surviving short stayers. In a small subgroup of patients with persistent critical illness, CSA negatively correlated with UCR at the time of the second scan. Hence, UCR could be a surrogate for muscle mass at that time.

Although the evidence is circumstantial, the physiological base for UCR, as a catabolic biomarker, is quite strong. Indeed, as catabolism likely increases ureagenesis, whereas progressive loss of muscle mass leads to decreased creatinine generation, the trajectories of urea and creatinine in catabolic state expectedly evolve in opposite directions. Hence, elevated UCR could be a physiologically plausible indicator of ongoing catabolism, and as such, be used as a signature for the catabolic state of persistent critical illness. Previous research showed similar time profiles of urea, creatinine, and UCR in

\section{Springer}


mixed ICU patients [8], suggesting that current findings are not specific to trauma patients.

The identification of UCR as a potential marker of critical illness-associated catabolism is highly relevant for future studies. Indeed, instead of merely investigating the end-product of catabolism (i.e., sarcopenia), future metabolic studies should consider monitoring UCR as a biomarker of the catabolic process itself. This may assist in identifying early effects of an intervention, before impact on muscle mass and function has become clear. Moreover, large observational studies could use UCR to gain insights into risk factors and potential therapeutic targets of critical illness-associated catabolism. In nutritional studies, however, it is important to consider that elevated UCR can result from metabolism of exogenous amino acids on top of endogenous catabolism [8,9].

Despite the potential role of UCR in future studies, clinical usability seems limited, as other factors may increase UCR independent of catabolism (Fig. 1). These include decreased effective blood volume induced by various factors such as diuretics, high dietary content of amino acids, and gastrointestinal bleeding $[8,10,11]$. Moreover, UCR may be altered by acute kidney injury
(AKI), depending on the relative increase of urea versus creatinine. In the study of Haines et al., severe AKI tended to lower UCR [7]. To some extent, these potential confounders may explain the considerable overlap in UCR between patients with short versus prolonged ICU dependency.

The use of renal function markers as indicators of low muscle mass, as the catabolic end-product, is not unprecedented. Previously, the sarcopenia index, calculated as the serum creatinine-to-cystatin $\mathrm{C}$ ratio, was described as a biomarker of decreased muscle mass in critically ill patients [12, 13]. Unlike urea and creatinine, however, cystatin $\mathrm{C}$ is usually not routinely measured. Hence, the use of UCR for estimating muscle mass may be more evident. However, caution is needed since urea is a product of active proteolysis, hence low UCR may not necessarily exclude low muscle mass. This contrasts with creatinine and cystatin $\mathrm{C}$, of which production rate is directly related to muscle mass and the number of nucleated cells, respectively. Moreover, the correlation between UCR and muscle CSA was only observed in a small subset of patients at one time point, which requires confirmation.

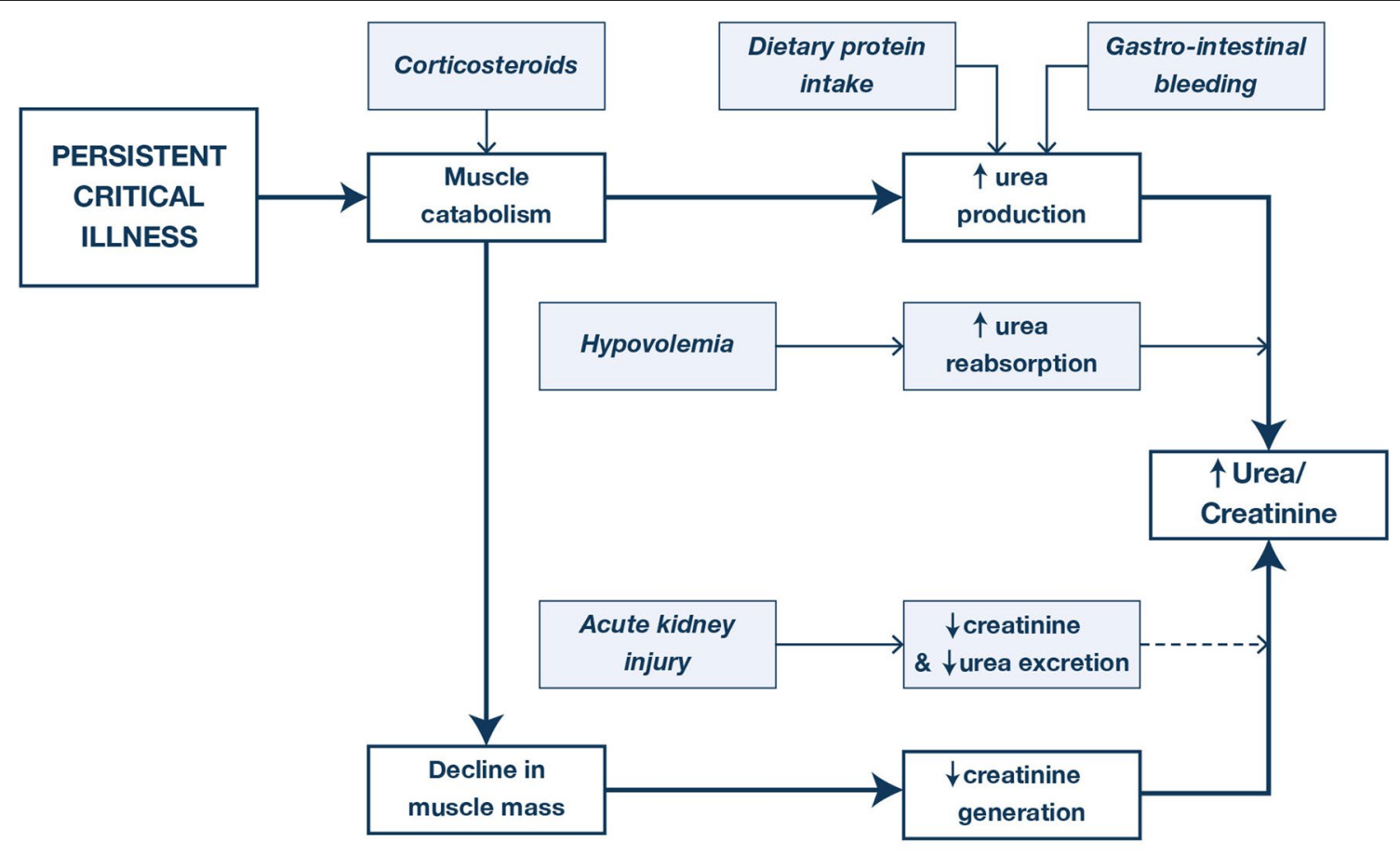

$\longrightarrow$ Main hypothesis, positive effect
$\longrightarrow \quad$ Potential confounder, positive effect
$---\rightarrow$ Potential confounder, negative effect

Fig. 1 Relationship between persistent critical illness, catabolic state, and urea-creatinine ratio. Potential confounders are depicted in gray 
Finally, another interesting finding is the progressive decrease of creatinine during critical illness, which is corroborated by other investigators [14]. This questions the accuracy of detecting AKI when solely relying on serum creatinine. Indeed, these data may indicate that during critical illness, such an approach may considerably underestimate the incidence of AKI [14].

In conclusion, Haines et al. identified UCR as a metabolic signature for the ongoing catabolic state characterizing persistent critical illness. Clinical usability of UCR appears to be limited by potential confounders that may increase UCR independent of catabolism. Whether UCR can act as marker of decreased muscle mass in persistent critical illness requires confirmation. Nevertheless, UCR could be a novel, readily available, and promising biomarker of catabolism in future epidemiological and clinical studies.

\section{Author details}

${ }^{1}$ Clinical Division and Laboratory of Intensive Care Medicine, Department of Cellular and Molecular Medicine, KU Leuven, Herestraat 49, 3000 Leuven, Belgium. ${ }^{2}$ Division of Nephrology and Hypertension, Division of Pulmonary and Critical Care Medicine, Department of Medicine, Mayo Clinic, 200 First Street Southwest, Rochester, MN 55905, USA. ${ }^{3}$ Medical Intensive Care Unit, Department of General Internal Medicine and Laboratory of Intensive Care Medicine, Department of Cellular and Molecular Medicine, KU Leuven, Herestraat 49, 3000 Leuven, Belgium.

\section{Acknowledgements}

JG holds a postdoctoral research fellowship from the Clinical Research and Education Council of the University Hospitals Leuven. GH holds a Fundamental Clinical Research fellowship from the Research Foundation-Flanders $(1805116 \mathrm{~N})$.

\section{Compliance with ethical standards}

\section{Conflicts of interest}

On behalf of all authors, the corresponding author states that there is no conflict of interest.

\section{Publisher's Note}

Springer Nature remains neutral with regard to jurisdictional claims in published maps and institutional affiliations.

Received: 5 September 2019 Accepted: 27 September 2019

Published online: 16 October 2019
References

1. Nelson JE, Cox CE, Hope AA, Carson SS (2010) Chronic critical illness. Am J Respir Crit Care Med 182:446-454. https://doi.org/10.1164/rccm.20100 2-0210Cl

2. Iwashyna TJ, Hodgson CL, Pilcher D, Bailey M, van Lint A, Chavan S, Bellomo R (2016) Timing of onset and burden of persistent critical illness in Australia and New Zealand: a retrospective, population-based, observational study. Lancet Respir Med 4:566-573. https://doi.org/10.1016/S2213 $-2600(16) 30098-4$

3. Kress JP, Hall JB (2014) ICU-acquired weakness and recovery from critical illness. N Engl J Med 370:1626-1635. https://doi.org/10.1056/NEJMra1209 390

4. Van den Berghe G (2016) On the neuroendocrinopathy of critical illness. Perspectives for feeding and novel treatments. Am J Respir Crit Care Med 194:1337-1348. https://doi.org/10.1164/rccm.201607-1516Cl

5. Hermans G, Van den Berghe G (2015) Clinical review: intensive care unit acquired weakness. Crit Care 19:274. https://doi.org/10.1186/s1305 4-015-0993-7

6. Ferrie S, Tsang E (2018) Monitoring nutrition in critical illness: what can we use? Nutr Clin Pract 33:133-146. https://doi.org/10.1177/088453361770631 2

7. Haines RW, Zolfaghari P, Wan Y, Pearse RM, Puthucheary Z, Prowle JR (2019) Elevated urea to creatinine ratio provides a biochemical signature of muscle catabolism and persistent critical illness after major trauma. Intensive Care Med. https://doi.org/10.1007/s00134-019-05760-5

8. Gunst J, Vanhorebeek I, Casaer MP, Hermans G, Wouters PJ, Dubois J, Claes K, Schetz M, Van den Berghe G (2013) Impact of early parenteral nutrition on metabolism and kidney injury. J Am Soc Nephrol 24:995-1005. https://doi. org/10.1681/ASN.2012070732

9. Gunst J, Vanhorebeek I, Thiessen SE, Van den Berghe G (2018) Amino acid supplements in critically ill patients. Pharmacol Res 130:127-131. https://doi. org/10.1016/j.phrs.2017.12.007

10. Macedo E, Mehta RL (2009) Prerenal failure: from old concepts to new paradigms. Curr Opin Crit Care 15:467-473. https://doi.org/10.1097/MCC.0b013 e328332f6e3

11. Srygley FD, Gerardo CJ, Tran T, Fisher DA (2012) Does this patient have a severe upper gastrointestinal bleed? JAMA 307:1072-1079. https://doi. org/10.1001/jama.2012.253

12. Kashani KB, Frazee EN, Kukralova L, Sarvottam K, Herasevich V, Young PM, Kashyap R, Lieske JC (2017) Evaluating muscle mass by using markers of kidney function: development of the sarcopenia index. Crit Care Med 45:e23-e29. https://doi.org/10.1097/CCM.0000000000002013

13. Barreto EF, Poyant JO, Coville HH, Dierkhising RA, Kennedy CC, Gajic O, Nystrom EM, Takahashi N, Moynagh MR, Kashani KB (2019) Validation of the sarcopenia index to assess muscle mass in the critically ill: a novel application of kidney function markers. Clin Nutr 38:1362-1367. https://doi. org/10.1016/j.clnu.2018.05.031

14. Schetz M, Gunst J, Van den Berghe G (2014) The impact of using estimated GFR versus creatinine clearance on the evaluation of recovery from acute kidney injury in the ICU. Intensive Care Med 40:1709-1717. https://doi. org/10.1007/s00134-014-3487-1 